\title{
How to Characterize Amorphous Shapes: The Tale of a Reverse Micelle
}

\author{
Christopher Gale, ${ }^{*} \dagger$ Mortaza Derakhshani-Molayousefi, ${ }^{*} \ddagger$ and Nancy E. \\ Levinger ${ }^{*} \dagger$ \\ $\dagger$ Department of Chemistry, Colorado State University, Fort Collins, Colorado 80523, USA \\ $\ddagger$ Department of Chemistry, University of Arkansas, Fayetteville, Arkansas 71656, USA \\ E-mail: cgale@colostate.edu; mderakhs@uark.edu; Nancy.Levinger@colostate.edu
}




\begin{abstract}
Aerosol-OT reverse micelles represent a chemical construct where surfactant molecules self-assemble to stabilize water nanodroplets $1-10 \mathrm{~nm}$ in diameter. Although commonly assumed to adopt a spherical shape, all-atom molecular dynamics simulations and some experimental studies predict a non-spherical shape. If these aggregates are not spherical, then what shape do they take? Because the tools needed to evaluate the shape of something that lacks regular structure, order, or symmetry are not well developed, we present a set of three intuitive metrics - coordinate-pair eccentricity, convexity, and the curvature distribution - that estimate the shape of an amorphous object and we demonstrate their use on a simulated Aerosol-OT reverse micelle. These metrics are all well-established methods and principles in mathematics, and each provides unique information about the shape. Together, these metrics provide intuitive descriptions of amorphous shapes, facilitate ways to quantify those shapes, and follow their changes over time.
\end{abstract}

\title{
1 Introduction
}

Since Dalton first proposed his atomic theory, the study of chemistry has been inextricably linked to the study of structure. ${ }^{1}$ Soon after Dalton, early organic chemists Kekulé and Couper discovered chemical bonding and invented the notation to represent molecules spatially. ${ }^{2}$ Seven years later, Kekulé proposed the first structure of benzene. ${ }^{3,4}$ By developing a robust understanding of structure, Kekulé and Couper paved the way for modern organic chemistry. ${ }^{5}$ Later, harnessing X-rays would allow researchers to characterize organic and inorganic crystals. ${ }^{6}$ This ability was used to determine the structure of penicillin, which showed researchers how to create a whole host of beta-lactam antibiotics and revolutionize medicine. ${ }^{7-9}$

Today, a wide range of experimental methods and theoretical frameworks exist to determine molecular, crystal, nanoparticle and meso-structures. However, these methods and 
frameworks only apply for highly organized, well-defined systems. Disordered and amorphous shapes have eluded description so far, despite the frequency with which such systems appear in nature. Polymers, biology, and soft materials, like self-assembled systems, frequently display disorder and adopt shapes that are not easily described with current theoretical frameworks. ${ }^{10}$

Aerosol-OT (AOT) reverse micelles are an excellent model system to explore amorphous shapes. These particles have been extensively studied and used because AOT creates remarkably robust aggregates with sizes closely related to the water:AOT ratio. ${ }^{11}$ A plethora of studies, from characterizing molecular behavior in nanoconfinement to templating nanoparticle synthesis ${ }^{12-22}$ make AOT reverse micelles exceptionally well characterized. Despite extensive use in research, there is continued debate on the shape of AOT reverse micelles. Much experimental work assumes that the aggregates are spherical, ${ }^{23-29}$ but other experimental work disagrees. ${ }^{30-33}$ While early simulations applied the same simplification of assuming a spherical micelle, ${ }^{34}$ the all-atom molecular dynamics simulations enabled by greater computing power have universally found the reverse micelles adopt some variation of a non-spherical shape. ${ }^{33,35-41}$ Simplifying assumptions are often made about AOT reverse micelles to build a framework for understanding the results. For example, Piletic, et al., used nonlinear IR spectroscopy to demonstrate two distinct water environments, "shell" and "core" water, calculated assuming a spherical shape. ${ }^{13}$ With the shape of these particles a matter of debate, new methods of investigating the shape are needed because the shape impacts how many studies and results are interpreted.

Some arbitrary and amorphous shapes can be replicated by a sum of spherical harmonics functions. ${ }^{42,43}$ To achieve the precision that defines the shape, these calculations involve hundreds of integrals over tens of thousands of points and require that the shape contain no hollow cavities or overhangs. While spherical harmonics can produce an exact facsimile of the shape and are quite convenient in several applications, such as X-ray tomography, they have no handle to develop intuition about the shape. Therefore, spherical harmonics are not 
flexible enough to accommodate many amorphous shapes that arise in chemical systems and tracking any individual or group of coefficients generated by the spherical harmonics model does not describe how the shape evolves over time or allow comparison.

To compare irregular, amorphous shapes in an ensemble, or to follow the evolution of a shape in time requires development of metrics that provide an accurate description of an arbitrary amorphous shape and capture their important and fundamental features. Here, we present a set of metrics - coordinate-pair eccentricity, convexity, and curvature - which are well-established in the field of mathematics, and provide an intuitive and meaningful description of the shape of amorphous objects. We apply these metrics to describe the amorphous shape of a simulated AOT reverse micelle and use the metrics to follow the evolution of shape over the course of the $100 \mathrm{~ns}$ simulation. We note that this analysis can be used to compare related amorphous shapes from a vast range of applications.

\section{Methods}

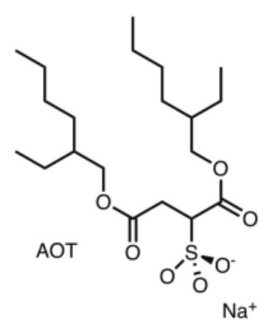

Figure 1: Structure of Aerosol-OT (AOT) surfactant.

To test and demonstrate the metrics of shape proposed here, this study uses the example of a reverse micelle system simulated with molecular dynamics. The micelle consists of isooctane, water, and the surfactant bis(2-ethylhexyl) sulfosuccinate, more commonly known as Aerosol-OT (AOT), shown in Figure 1. The exact aggregation number - the number of surfactant molecules per micelle - for an AOT reverse micelle is not precisely known, but the Abel lab provides reasonable estimates based on experimental data. ${ }^{35,44,45}$ These estimates agree with the most extensive computational search for the aggregation number of AOT 
reverse micelles performed to date. ${ }^{41}$ We use those numbers for our simulation and therefore the simulation consists of 1,500 isooctane molecules, 210 water molecules, and 42 AOT molecules. Isooctane and AOT were modelled using the CHARMM36 force field, ${ }^{35,46}$ while water was modelled with the TIP3P force field, ${ }^{47}$ in keeping with the Abel groups specifications and previous all-atom molecular dynamics simulations of reverse micelles. ${ }^{33,35,37-41,44}$

The simulations were carried out using the 2019 series GROMACS package. ${ }^{48-51}$ The system was packed into a spherical micelle using Packmol. ${ }^{52}$ The system was minimized by steepest descent to remove any overlapping molecules from the packing. Equilibration was performed in several steps to ensure that the shape of the micelle was not influenced or biased by the starting structure, as detailed in Table 1. The equilibration steps were designed to maintain position restraints on the core while allowing the system to relax from the outside in. The system was allowed to run for another 9 ns with no position restraints on any component to complete the equilibration. All equilibration steps were performed with an NPT ensemble using a V-rescale thermostat ${ }^{53}$ and Berendsen barostat ${ }^{54}$ with a 1 fs step size. The data presented here was collected from a production run of $100 \mathrm{~ns}$ using the NPT ensemble with the Nose-Hoover thermostat ${ }^{55,56}$ and Parrinello-Rahman barostat ${ }^{57,58}$ and a 0.5 fs step size. Both equilibration and production runs were held at 1 bar and $298 \mathrm{~K}$.

\section{Table 1: Equilibration Schedule for Reverse Micelles}

\begin{tabular}{ccccc}
\hline \multirow{2}{*}{$\begin{array}{c}\text { Step } \\
\text { Time (ns) }\end{array}$} & $\begin{array}{c}\text { Total Time } \\
\text { Equilibrated (ns) }\end{array}$ & Water & AOT & Isooctane \\
\hline 0.2 & 0.2 & 1000 & 1000 & 1000 \\
0.5 & 0.7 & 1000 & 1000 & 0 \\
0.1 & 0.8 & 1000 & 500 & 0 \\
0.1 & 0.9 & 1000 & 0 & 0 \\
0.1 & 1.0 & 500 & 0 & 0 \\
9.0 & 10.0 & 0 & 0 & 0 \\
\hline
\end{tabular}

Custom Python code was written to perform all shape analyses described here and is being released with the SI. The core workflow is to first create a Willard-Chandler surface of the micelle. ${ }^{59,60}$ This produces a triangulated mesh representation of the micelle on which the analyses can then be performed. ${ }^{61}$ The code uses the MDAnalysis package ${ }^{62,63}$ for ma- 
nipulation of the molecular dynamics trajectory and the PyVista package ${ }^{64}$ for manipulation of the mesh. For all of the analyses shown here, the water, sodium counterions, and succinate headgroup of AOT were considered as the micelle surface. The choice of surface almost certainly impacts the results and a comparison of different choices likely holds valuable information about the system. However, these types of considerations are beyond the scope of this paper and will be explored in a subsequent paper.

\section{Metrics of Shape}

\subsection{Coordinate Pair Eccentricity}

In mathematics, eccentricity is a number that uniquely characterizes the shape of a conic section. ${ }^{65}$ It distinguishes between all conic sections and provides ellipses and hyperbolae with unique identifiers, even within their respective classifications. ${ }^{66}$ Focusing only on the closed form conic sections, that is on ellipses, eccentricity is defined as:

$$
e=\sqrt{1-\frac{b^{2}}{a^{2}}}
$$

where $e$ is the eccentricity, $a$ and $b$ are the major and minor semiaxes, respectively. To adapt this definition to characterizing physical objects, which are necessarily three-dimensional, the moments of inertia have been used historically. ${ }^{35,37,38}$ In this view, an ellipsoid of equivalent mass but constant density is created by equating the principal moments of inertia of the object to the well-known equations for the moments of inertia for an ellipsoid of equivalent mass. ${ }^{67}$ The addition of a new dimension also means that a new semiaxis must be introduced, 
$c$, to transition from an ellipse to an ellipsoid.

$$
\begin{aligned}
& A=\frac{M}{5}\left(b^{2}+c^{2}\right) \\
& B=\frac{M}{5}\left(a^{2}+c^{2}\right) \\
& C=\frac{M}{5}\left(a^{2}+b^{2}\right)
\end{aligned}
$$

In this formulation, $M$ is the mass of the object, $A, B$, and $C$ represent the magnitudes of the three principal moments of inertia, and $a, b$, and $c$ represent the semiaxis lengths. Each principal moment of inertia shares a vector direction with the semiaxis of the same letter, e.g. $A$ and $a$ share the same vector direction. Semiaxis lengths are easily determined from these equations using a linear algebra approach. Eccentricity, defined in Equation 1, becomes imaginary if $b \geq a$. We have adopted the convention that $a \geq b \geq c$, which agrees with past literature and mathematical standards. Note that using this convention also implies that $A \leq B \leq C$

Ellipsoids are generally sorted into one of three broad categories: spheres, oblate ellipsoids, and prolate ellipsoids. ${ }^{68}$ Applying an eccentricity value to describe a three dimensional object using the standard, mathematical definition in Equation 1, becomes problematic, because the equation describes conic sections that are necessarily two dimensional. Most commonly, only two of the three semiaxes are selected as input; however this leads to an inherent loss of information. Regardless of which two semiaxes are chosen, at most only two of the categories of ellipsoids can be distinguished. To characterize a three dimensional ellipsoid using eccentricity that does not lead to a loss of information requires use of all three semiaxes to report two different values of eccentricity. We introduce this concept as coordinate-pair 
eccentricity (CPE), as given in Equations 3.

$$
\begin{aligned}
& e_{a b}=\sqrt{1-\frac{b^{2}}{a^{2}}} \\
& e_{a c}=\sqrt{1-\frac{c^{2}}{a^{2}}}
\end{aligned}
$$

Here, $e_{a b}$ and $e_{a c}$ are the pair of values that constitute the CPE. We chose these values as they allow us to differentiate between all three categories of ellipsoids. A spherical ellipsoid is typified by the relation $a \approx b \approx c$, while a prolate ellipsoid is typified by $a>b \approx c$, and an oblate ellipsoid is typified by $a \approx b>c$. In more familiar terms, a prolate ellipsoid is a roughly cigar shaped object while an oblate ellipsoid resembles a disk or an M\&M candy. $e_{a c}$ differentiates between a spherical object and an eccentric ellipsoid, but not between whether that eccentric ellipsoid is prolate or oblate ellipsoids because $a>c$, in both cases. By including $e_{a b}$, this redundancy is removed and all three cases may be differentiated.

\subsection{Convexity}

The second metric characterizes the deviations from an ideal, convex shape. For a shape to be convex, the line connecting any two points on the surface must be completely contained by the shape (assuming a topological ball, i.e. no cavities or hollow spaces inside). ${ }^{69} \mathrm{~A}$ convex hull is the smallest convex object or set that contains the shape of interest. Many common shapes are convex, including squares and circles in two dimensions, and spheres and dodecahedra in three dimensions. However, it is easy to find many shapes that do not meet this definition such as a star (formally, an augmented pentagon or hexagon). Convexity quantifies how far an arbitrary shape is from being convex.

The convexity can be defined in a number of ways. ${ }^{69}$ However, the more complex definitions are designed to provide usable values even in situations such as infinitely thin rods, spikes, or trenches that cannot be realistically obtained for a shape derived from real, finite objects made up of atoms and molecules. Therefore, we have chosen the computationally 
simple and efficient definition:

$$
\Xi=\frac{\text { Volume }(S)}{\operatorname{Volume}(C H(S))}
$$

where we use $\Xi$ to represent the convexity, $S$ denotes the shape of interest, Volume() denotes the volume of the argument, and $C H()$ denotes the convex hull of the argument. Once again, the convex hull is the smallest possible shape that circumscribes the shape of interest and is convex. As shown by Equation 4, convexity has a range of $(0,1]$. When $\Xi=1$, the shape and its convex hull are identical, and therefore have the same value. Similarly, a value of 0 cannot be realistically obtained for real, finite objects as this would imply that the shape has zero volume and comprises vanishingly thin rods.

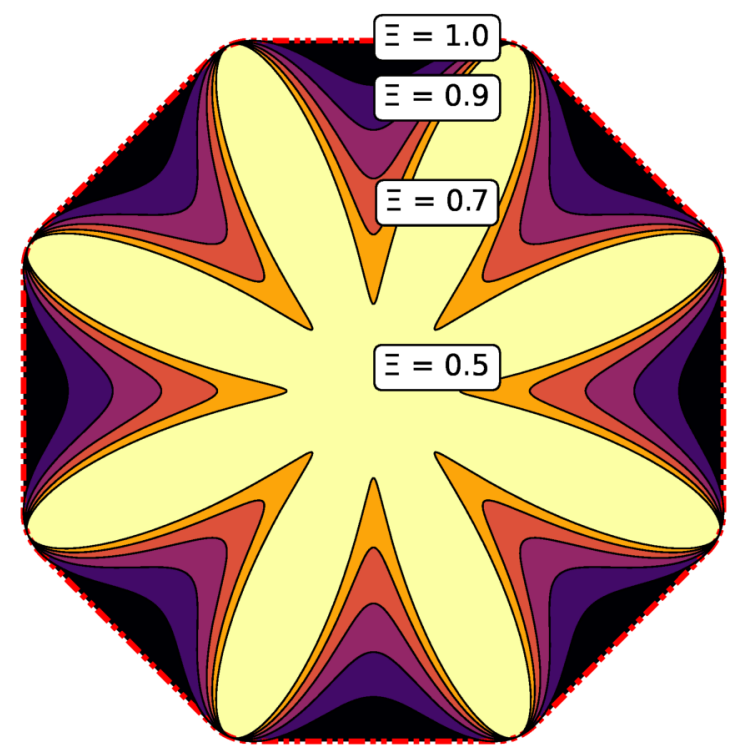

Figure 2: Example figure of convexity in two dimensions. Each color represents a different shape with one tenth less convexity. The convex hull for all of the shapes is shown as a red, dashed line.

A visual reference for the physical meaning of different convexity values is given in Figure 2, showing convexity using area for a 2D representation, rather than volume as defined in Equation 4. The definition of convexity chosen here, in Equation 4, conveniently allows convexity to be thought of as the shape's fraction of area relative to its convex hull. For a 
completely convex shape, $\Xi=1$, and $\Xi$ will get successively smaller as more area is "removed" from the shape. For example, the yellow shape shown in Figure 2 has a convexity of 0.6 because it has $60 \%$ of the area of its convex hull.

\subsection{The Curvature Distribution}

The third shape metric presented is the curvature distribution. Curvature of a two dimensional curve (of the form $y=f(x)$ ) is closely related to the second derivative of the curve and can be most intuitively defined as the inverse of the radius of an osculating circle, which is a circle that closely approximates the curve at that point. ${ }^{70}$ An example of an osculating circle is provided in the SI (Figure S3). For a three dimensional surface, curvature is no longer a single value, but pair of values known as the principal curvatures, computed along orthogonal vector directions. ${ }^{71}$ There are two primary ways in which these principal curvatures can be utilized, that is mean and Gaussian curvature, given in Equations 5a and 5b, respectively.

$$
\begin{aligned}
& \kappa_{m}=\frac{k_{1}+k_{2}}{2} \\
& \kappa_{G}=k_{1} k_{2}
\end{aligned}
$$

Here $k_{1}$ and $k_{2}$ are the principal curvatures. In Equation $5 \mathrm{a}, \kappa_{m}$, is the mean curvature and in Equation 5b, $\kappa_{G}$, is known as the Gaussian curvature. ${ }^{71}$ Note that the curvature is defined to be in the direction of the osculating circle describing it (see Figure S3 for an example). As a matter of convention, the principal curvature is considered positive if the surface is curved toward the interior of the shape and negative if it is curved away from the shape.

As the literal average of principal curvatures, mean curvature represents the average trend

of the surface at the point where it is computed. ${ }^{72}$ A positive mean curvature indicates that the surface curves toward the center of the shape at that point. It is possible to have principal curvatures of opposite signs, but the positive principal curvature must dominate for the mean 
curvature to be positive, and so the surface is mostly positively curved. Gaussian curvature, on the other hand, indicates the nature of the surface at a particular point. A positive Gaussian curvature means that both principal curvatures have the same sign, either positive or negative, and the surface at that point is considered elliptic. ${ }^{72}$ This is the type of surface found at every point on an ellipsoid, where both vectors of principal curvature curve in the same direction. Where the Gaussian curvature is negative, one principal curvature must be positive while the other is negative leading to a hyperbolic point. ${ }^{72}$ A hyperbolic point is most recognizable as the type of surface found at a saddle point.

\section{Results and Discussion}

\subsection{Coordinate-Pair Eccentricity}

Extending the classical definition of eccentricity ${ }^{66}$ to the third dimension allows us to describe the distribution of mass within an amorphous shape by differentiating between spherical, prolate and oblate ellipsoids. However, the use of eccentricity is not the only method which has been used to characterize shape in this way. Some groups use a variation on eccentricity usually represented as $\eta_{1}$ and $\eta_{2} \cdot{ }^{36,73,74}$ We explore the relationship between the two metrics in detail in the SI, but believe that CPE represents a much more straightforward and easy to use metric. Figure 3 presents the CPE over time for the reverse micelle. The "standard" definition of eccentricity, ${ }^{66}$ based on the study of $2 \mathrm{D}$ conic sections and frequently used for these reverse micelles, is the same as CPE's $e_{a c}$ parameter shown in Figure 3d. An increase in $e_{a c}$ correctly determines that the micelle is no longer spherical, but as shown in Figure 3a, a change in $e_{a c}$ cannot distinguish between prolate and oblate ellipsoids. The addition of $e_{a b}$ (Figure 3c) distinguishes between the two basic ellipsoids.

CPE is best thought of as the zeroth order approximation of shape. The semiaxes from which eccentricity is computed arise directly from the object's moments of inertia. Although the underlying shape is often significantly more complex, the remaining metrics presented 
a

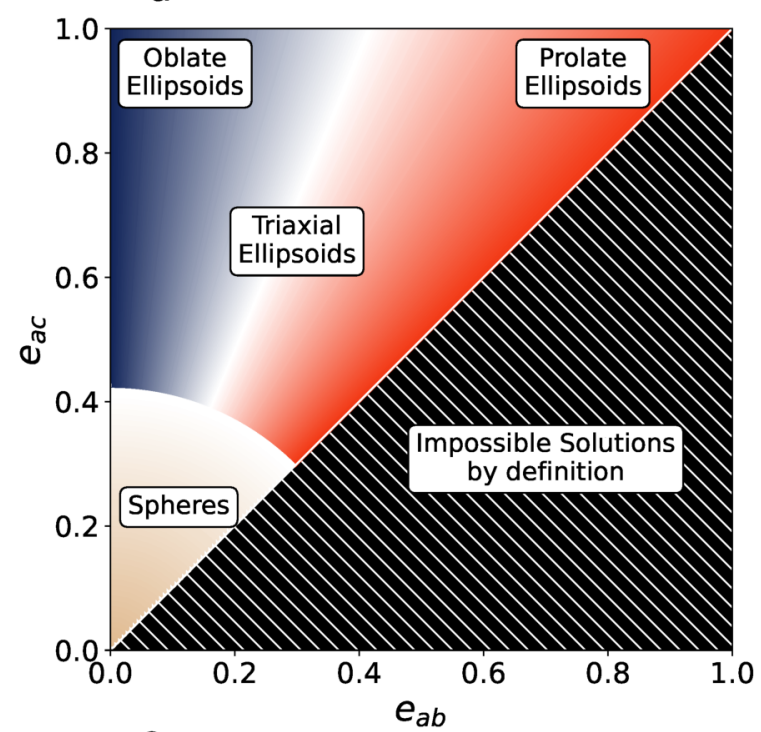

C

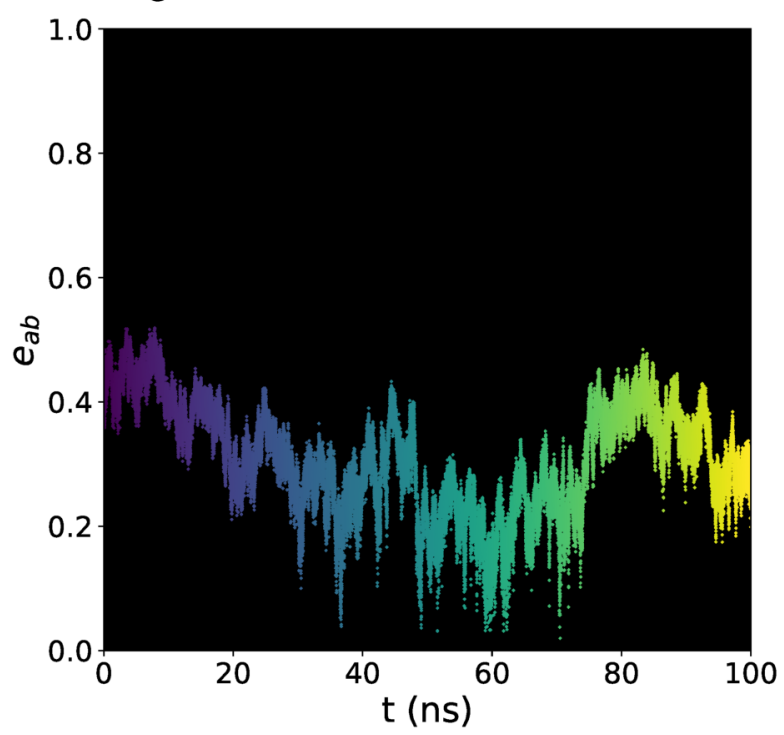

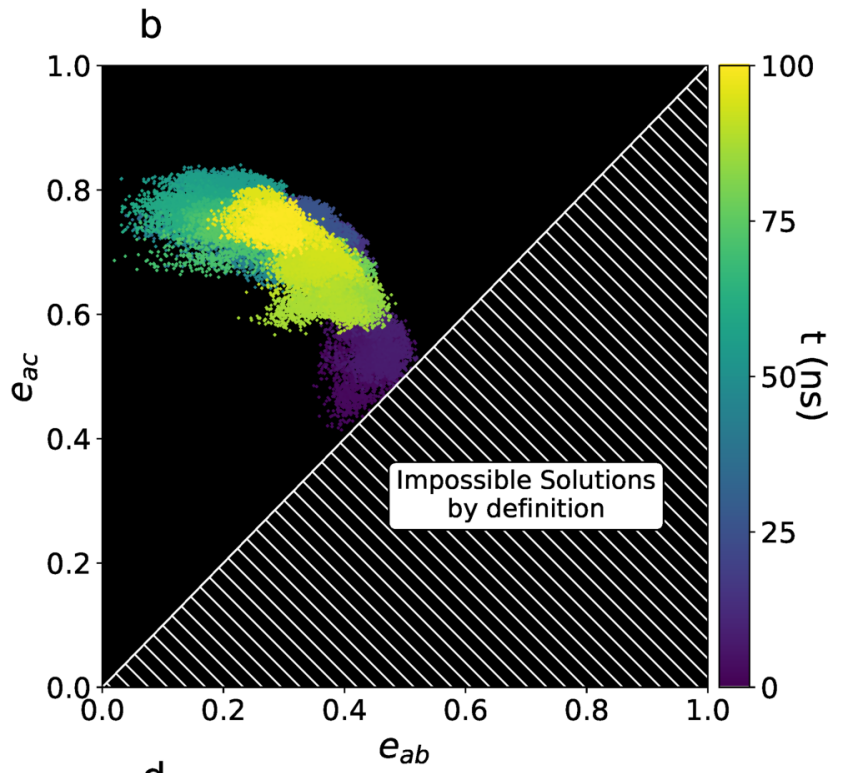

d

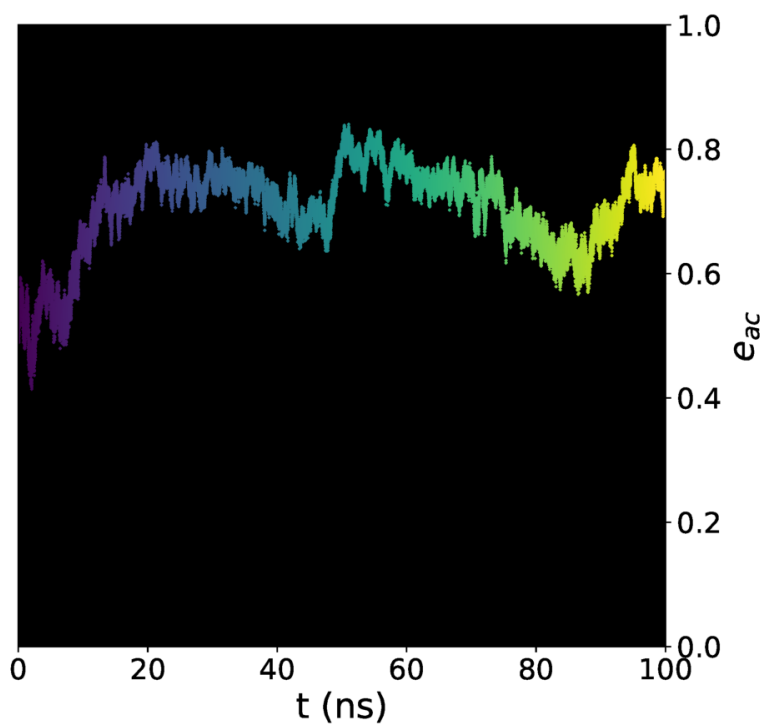

Figure 3: (a) Guide to coordinate-pair eccentricity: $e_{a c}$ compares the largest and smallest semiaxes; $e_{a b}$ compares largest and intermediate axes. The colors indicate approximate regions where different basic ellipsoids occur. These areas are approximate because there is no formal definition delineating, e.g., when a mostly spherical object stops being spherical. Solutions in the hatched area contradict the assumption that $\mathrm{a}>\mathrm{b}>\mathrm{c}$. (b) Coordinate-pair eccentricity of the micelle. Time is displayed as a color according to the bar at right. (c) $e_{a b}$ versus time. (d) $e_{a c}$ versus time.

here are of little use without this basic model to build upon. None of the previous work, either simulated or experimental, has been able to characterize whether reverse micelles tend to be more prolate or oblate ellipsoids. ${ }^{23-33,35-41}$ The best descriptions available are images 
of simulated micelles, which appear largely prolate. ${ }^{37,41}$ As Figure 3 shows, the micelles have a surprising level of diversity of shape, showing fluctuations between being a predominantly prolate and oblate ellipsoid which have not previously been quantified.

Because it is only a very rough approximation of shape, the CPE has limitations. It is a starting place rather than a complete description of the shape by itself. For example, CPE would classify a spiral shape, possibly encountered in polymer or biological chemistry, as a prolate ellipsoid given the distribution of mass and relative aspect ratio. Likewise, CPE would classify a U-shaped object, such as a kielbasa sausage or the micelle image in the green box in Figure 4 as an oblate ellipsoid, while human intuition would interpret these shapes as folded prolate ellipsoids. Although this tendency may occasionally violate intuition, it is an accurate description of the mass distribution, and more importantly, quantifiable and reproducible.

In addition, sensitivity to changes in eccentricity is not uniform across all values. The CPE response depends on the initial value; for smaller initial values of eccentricity, unit changes in semiaxis length lead to larger changes to eccentricity. This effect is clearly displayed in Figure $3 \mathrm{c}$ between $30-70$ ns where $e_{a b}$ has, on average, a significantly smaller value than anywhere else in the simulated time. Accordingly, $e_{a b}$ appears to have significantly larger fluctuations than $e_{a c}$. This can be explained by the significantly increased response of eccentricity to the same changes in semiaxis length when the eccentricity is small. A plot of the eccentricity as a function of semiaxis length is provided in the SI (Figure S4) to further demonstrate this point.

\subsection{Convexity}

CPE provides a good starting place for describing amorphous objects, but there are vastly different shapes that yield the same CPE values. For example, any basic regular polyhedron, such as a cube, is indistinguishable from its augmented variation that replaces the faces of the polyhedron with pyramids whose height could be either positive or negative, 
effectively creating "spiky" shapes. ${ }^{75,76}$ Regardless of the augmentation used, the CPE value of any regular polyhedron will be $(0,0)$ and classified as a perfect sphere. To differentiate between such objects and further characterize an amorphous shape, we propose convexity as a second metric. ${ }^{69}$ Defined in Section 3.2, convexity is the difference between the object and its convex hull. The convexity provides complementary information to CPE. Fluctuations in eccentricity predominantly reflect how the micelle deforms in a direction tangential to its surface. For example, a movement that stretches the shape from a sphere into a prolate ellipsoid alters the distribution of mass and therefore CPE. While there are movements, such as bends, which both metrics can detect; fluctuations in convexity tend to reflect motion normal to the reverse micelle surface because these irregularities alter convexity but do not necessarily change the CPE.

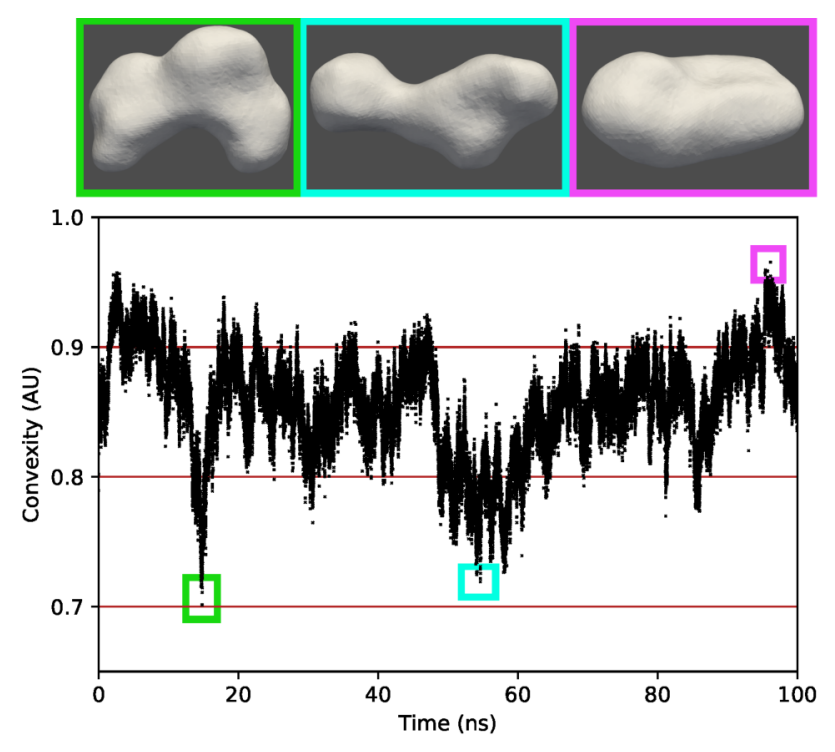

Figure 4: Convexity of the reverse micelle over the time of the full simulation. Representative images of reverse micelles at select times indicated by highlighted boxes appear above the convexity data.

The convexity of the simulated reverse micelle is presented in Figure 4, along with example images in several key portions of the simulation. These data demonstrate two key points. First, the micelle never exhibits an irregularity that removes more than $30 \%$ of the volume from the convex hull. Even in the most extreme case highlighted in green where a fold 
appears to have formed, the convexity only drops to a value of 0.7 . Second, the convexity never reaches a value of one, showing that the reverse micelle is never completely convex and always has some irregularities. The conclusion is that the micelles are largely convex but invariably include numerous bumps and divots on the surface.

In general, we observe two different types of changes to the shape that contribute to the fluctuations in convexity. Fast, low amplitude fluctuations arising from small movements normal to the surface, likely from the surface sinking or growing locally to form a divot or bump. We also observe higher amplitude and typically slower fluctuations arising from larger changes to shape. The image highlighted in the green box in Figure 4 demonstrates such a slower fluctuation and has reduced convexity compared to other points. Over time, this fold disappears and a new general shape forms, that is a bubble connected by a thin neck to the remainder of the micelle in a dumbbell-type configuration, as highlighted in the cyan box. These irregularities can also disappear to form a highly convex shape, such as is the image in the magenta box.

\subsection{Curvature Distribution}

We require one more metric to characterize the particle shape for one simple reason: CPE and convexity considered alone cannot differentiate between regular geometric shapes such as a sphere and a cube. The CPE would indicate both to be perfectly spherical and both shapes are also fully convex. The key to differentiating these shapes is their curvature distributions, presented as a probability density function (PDF). Recall that, as defined in Section 3.3, mean curvature shows the direction and magnitude of the curvature of the surface at a point; and that positive mean curvature values indicate that the surface curves toward the interior of the shape. Gaussian curvature provides information about the nature of the surface. A positive Gaussian curvature indicates an elliptic point while negative Gaussian curvature indicates a hyperbolic point. ${ }^{72}$ The surfaces of a sphere and cube both lack saddle points, so the Gaussian curvature is always positive. Thus we consider only mean curvature 
to compare the forms. A sphere has constant, positive mean curvature creating a probability density function that is a delta function at the value for its size. A cube has only flat faces and the joints between them leading to a sum of two delta functions, renormalized to one, with a distribution at zero for the flat faces and a distribution at infinity for the edges and corners.

In the case of a general amorphous shape, a priori, the distribution is expected to represent more than just a single value. If the smallest value of principal curvature over the entire surface is zero, then the shape must be fully convex, as demonstrated by Figure 2. Therefore, if the convexity is less than one, then divots exist somewhere on the surface and these must have negative principal curvature(s) at some point.

We calculate the curvature value at each point on the surface of the triangulated mesh of the reverse micelle for each time point in the simulation. To reduce the data to a viewable set, we generate a histogram of curvature values at each time point. The exact number of points at which curvature is computed varies, with typical values being between 1-3,000 points. Because the reverse micelle shifts from a predominantly prolate ellipsoid to a more oblate ellipsoid over the course of the simulation, Figure 5 presents examples of the mean curvature distribution at time points from the beginning and end of the simulation to sample varied cases.

Figure 5 provides several comparisons to guide its interpretation. The mean curvature distribution fits well to the sum of two Lorentzians, which we compare to the curvature distribution of an ideal ellipsoid with the same eccentricity and dimensions as the micelle at the selected time points. The expected curvature for a sphere of the same size as the micelle is provided a simple reference for size-independent comparisons, because curvature is an extrinsic value. The curvature distributions are highly characteristic of the shape, with distinct patterns for both prolate and oblate ellipsoids (see the SI for additional patterns of ideal ellipsoids, Figure S5). Curvature distributions for the ideal ellipsoids tend to display two sharp peaks, as seen in Figure 5, but the exact shapes and locations vary. However in 

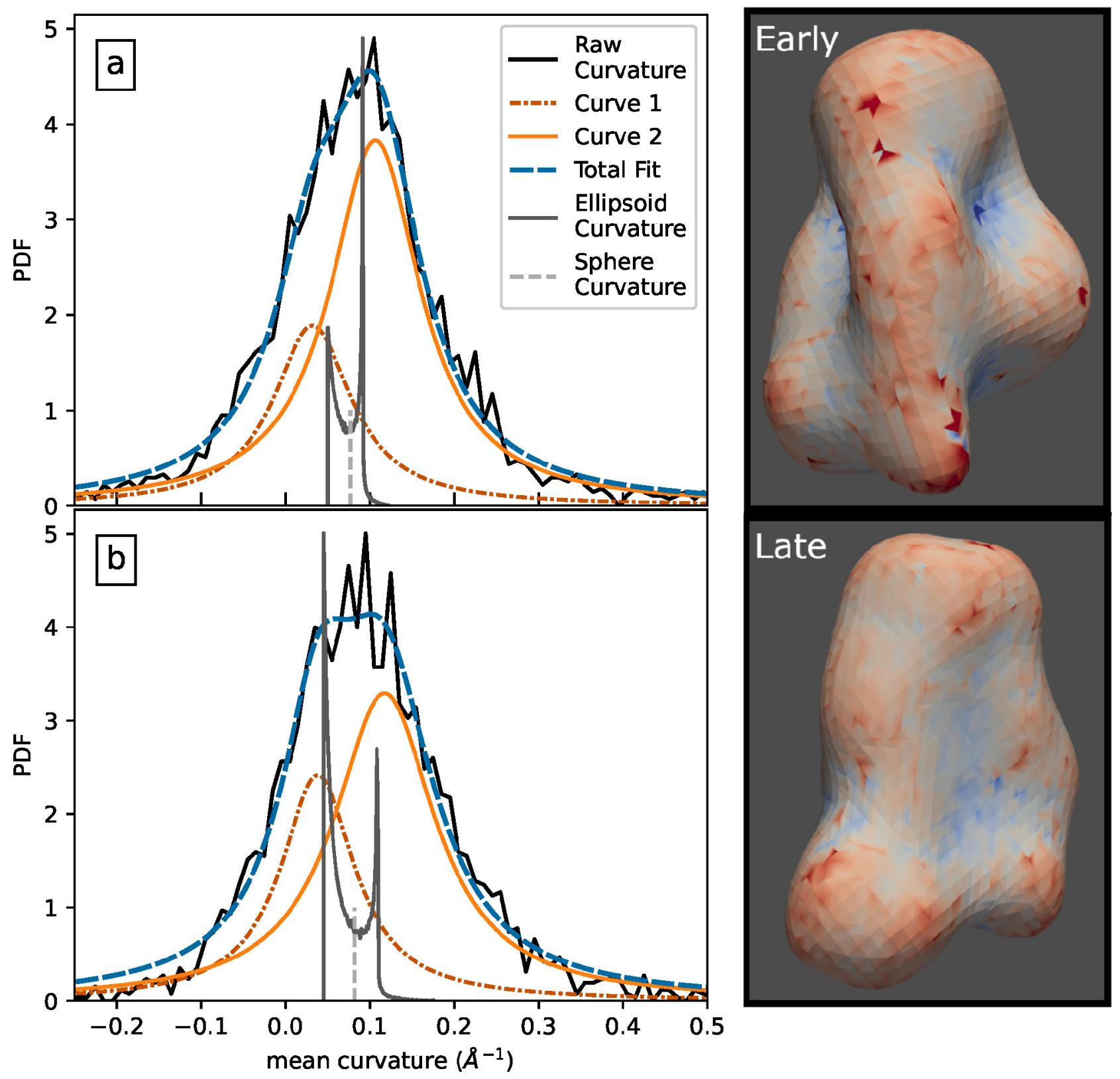

Figure 5: The curvature distribution of two random frames from the beginning (a) and end (b) of the trajectory (Legend: Raw Curvature). Each curvature distribution is fitted to the sum of two Lorentzians (Legend: Curves $1 / 2$, and Total Fit). The distribution for the ellipsoid computed from the moments of inertia is also plotted, renormalized so it fits on the graph (Legend: Ellipsoid Curvature). The expected distribution for a sphere of this size is shown to provide a reference for an object this size (Legend: Sphere Curvature). Images on the right show the micelle at these times, colored by curvature with positive values in red.

all cases, scaling the semiaxes to be larger shifts the observed peaks to the left- towards smaller, positive values - and reduces the domain while maintaining the pattern for that 
eccentricity. Reducing the sizes of the semiaxes shifts the peaks to the right and increases the domain.

As Figure 5 shows, the peak maxima of each fitted Lorentzian appears near a peak in the curvature distribution of the ideal ellipsoid. This suggests that the fitted Lorentzians represent the two peaks from the ideal ellipsoid curvature distribution, albeit with significant broadening. There are several sources of broadening relative to an ideal ellipsoid. First, with bumps and divots on its surface, the reverse micelle is not a true ellipsoid. If we assume that the observed shape is an ellipsoid that has been subsequently distorted, then creating a divot on the surface removes some population from the curvature value at that place and creates population on the left side of the distribution - at negative values. Since the most likely curvature values for a randomly chosen portion of the surface are the peak values of the distribution, the creation of divots has a "Robin Hood" effect that will tend to decrease the peak intensities and raise the left side of the distribution. The boundary points between a divot's negative curvature and rest of the surface creates flat regions of zero curvature at the interface that further broaden the distribution - the divot removes a curved portion to create the flat interfacial region - and smooth the distribution between the left side and the peak values. An analogous process occurs for bumps on the surface, operating on the right side of the distribution, removing from the peaks, increasing, and smoothing the population on the right wing of the distribution.

A second broadening mechanism arises because the curvature is computed numerically. For a well-defined function, the curvature can be calculated analytically and is exact. However, on a Delauney triangulated surface, there is no real curve. ${ }^{61}$ There are only points connected by straight lines approximating a curved surface, and an algorithm must be used to estimate this curvature. The algorithm we use to calculate curvature (a part of the PyVista package ${ }^{64}$ ) sometimes creates erroneously large values that shift population from the peak areas and move it toward the extreme tails of the distribution. A more advanced algorithm may reduce this error, but will not eliminate it entirely. This has the effect of 
broadening the distribution symmetrically. These overestimated values appear as the deeply colored spots on the representative micelle surfaces shown in Figure 5 that are clearly out of place with the surrounding values.

The bump-and-divot broadening described here does not act symmetrically on the peak in the same way that common sources of broadening, such as homo- or heterogeneous spectroscopic broadening, do. Rather than modifying a well-defined spectroscopic transition, the broadening mechanism described here quite literally erases the imaginary "previous" values and replaces them with other values. This literal substitution, rather than modification, of values can explain why the fitted Lorentzians bracket the peaks of the curvature distributions of the ideal ellipsoids.

Having described, in Figure 5, the curvature distributions for two select times, Figure 6 presents the mean and Gaussian curvature distributions over the full simulation. The mean curvature shows a relatively broad peak that is roughly symmetric and centered on a positive value. In contrast, the Gaussian curvature, shows a sharp, asymmetric peak with significantly more positive Gaussian curvature than negative, but the median value is almost exactly zero, skewing only slightly positive. The micelle is a topological ball, so even with its various irregularities, the most likely shape should have more elliptic than hyperbolic points, which readily explains the asymmetry.

The most striking feature of Figure 6, is how little it changes over time. Figure 5 suggests that the mean curvature predominantly follows the curvature distribution of an ideal ellipsoid, with some broadening indicating the formation of bumps and divots. However, over the course of the simulation, there is no evidence of a significant broadening, narrowing, or shift in peak position of the overall distribution. This constancy in curvature is in contrast to the concerted shift in CPE observed in Figure 3. We attribute this stability to two factors. First, the simulation ensures that volume of the micelle is constant. Constant volume implies that the curvature must revolve around some similar set of values because curvature is an extrinsic value. Drift toward either larger or smaller curvature values would 

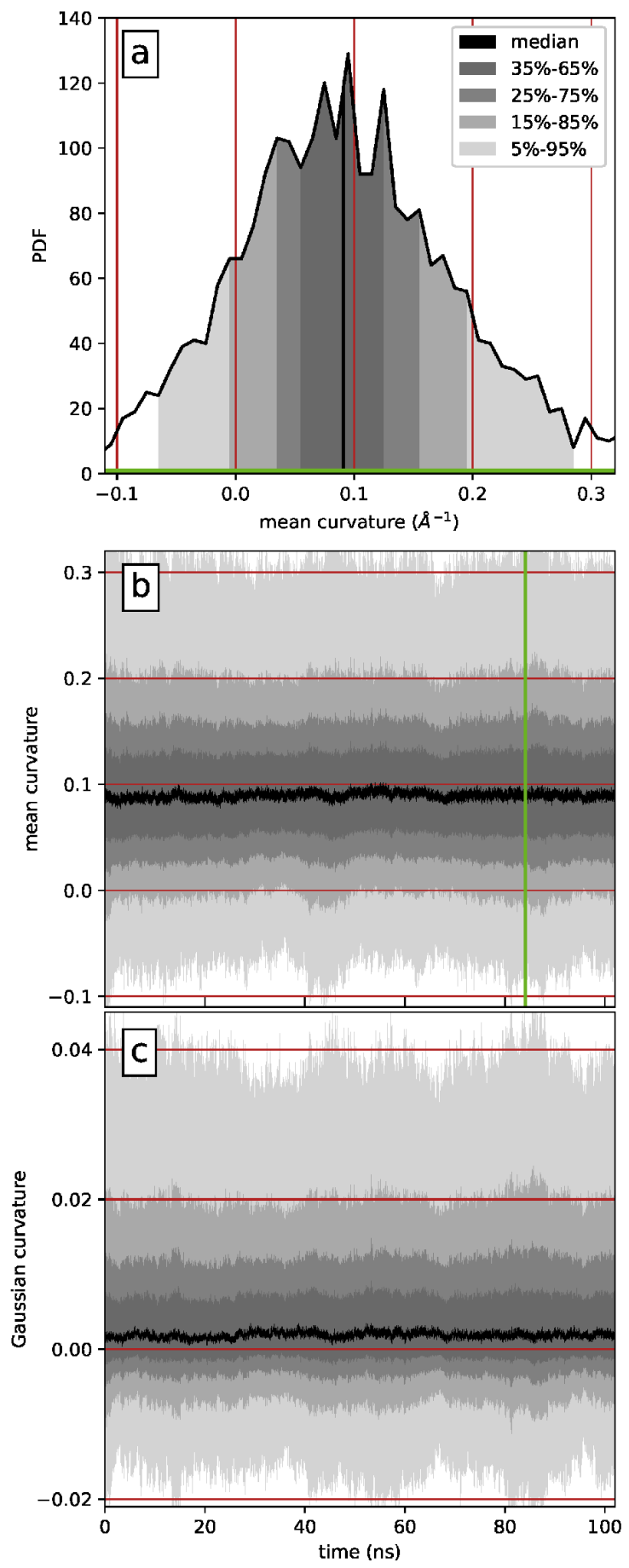

Figure 6: (a) Mean curvature distribution from the early frame of Figure 5. The black vertical line is the median of the distribution. The other shades of gray represent percentile ranges from the median: from darkest to lightest, $30 \%, 50 \%, 70 \%, 90 \%$. (b) Mean curvature distribution over all times. The example in part (a) represents a single slice of the data presented here, shown as a green line. (c) Gaussian curvature distribution over all times. 
indicate a change in size, which is impossible for a system of set stoichiometry such as the simulated micelle. In contrast, $\mathrm{CPE}$ is an intrinsic value, so its concerted shift is not reflected in the curvature distribution. A constant volume also means that any deformation to one side of the shape must move the displaced volume somewhere else, creating an endless supply of roughly similar bumps and divots. Second, it is likely that the forces acting on the micelle are relatively constant. If the micelle has been properly equilibrated, then the entire simulation exists in the same potential energy minimum, producing roughly equivalent forces at all times. We see no drift in the eccentricity or convexity to indicate the micelle is still migrating to a minimum, and we match or exceed the equilibration times for any simulated reverse micelles in literature, ${ }^{33,35-37,40,41}$ so we assume that this condition has been met. Then, assuming this static force acting on the micelle, the forces acting on the micelle must be responsible for the creation of the imperfections observed. Therefore, the dissipation of one imperfection must create the same sort of strain somewhere else, leading to the formation of a new imperfection. With constant volume ensuring the distributions remain centered on a constant value, and a mostly constant set of imperfections leading to constant broadening, the curvature distribution on whole remains quite stable.

\section{Applications, Limitations, and Conclusions}

We propose three metrics - coordinate pair eccentricity (CPE), convexity, and curvature distribution - to characterize and quantify amorphous shapes, particularly those found in chemistry. With these parameters, researchers can describe shapes that have until now relied on visual inspection. These metrics also allow us to compare similar systems quantitatively, track changes and fluctuations, and even compute characteristic times for these fluctuations. Most importantly, although our code is designed to work with a molecular dynamics sim-

ulation, as long as the system can be converted to a Delauney triangulated mesh, ${ }^{61}$ this analysis can be performed on absolutely anything, including systems outside the purview of 
chemistry.

We envision these metrics being used in a variety of ways in the study of reverse micelles. For example, we have found it exceptionally curious that the micelle has the same consituents everywhere, presumably leading to the same interactions everywhere, and yet adopts a shape where certain regions are highly curved and others are hardly curved at all. This suggests just the opposite of our naïve analysis: that the interactions are not the same everywhere. While in this study we have presented the reverse micelle only as an example system, we hope that a more thorough investigation into the shape of these reverse micelles will provide further information about this mystery. We have also noticed that in the work of Eskici and Axelsen to find the aggregation number of AOT reverse micelles via computed interaction energies, ${ }^{41}$ the aggregation number appears to also impact the shape quite significantly. Therefore, it may be possible to greatly simplify this work and find the aggregation number of a variety of different sizes of AOT reverse micelles in a fraction of the computational time via a shape analysis. These shape metrics could also be used to create a large number of simulated small-angle X-ray scattering curves with, e.g., a Monte Carlo approach to refine experimental interpretation and reconcile experimental work with computational work in regards to the shape of the micelles or other soft materials. We also hope that these metrics will provide a common ground for comparison between different studies and verification of new work against old work; such as comparison between the work of Abel, et al. ${ }^{35}$ the work of Martinez, et al. ${ }^{37}$ and the work of Eskici and Axelsen, ${ }^{41}$ who have all used slightly different aggregation numbers and water:AOT ratios which undoubtedly impacted the shape of the micelles.

Although these metrics provide robust characterization, they are non-unique. Thus, it is not possible to reproduce a single, unique shape purely from these metrics. Although the realm of amorphous shapes is immense, the metrics we describe here have an exceptional ability to distinguish between similar shapes, and are great tools for comparative analysis. Here we take a brief inventory of some situations where these metrics may or may not work. 
Just like in our example system, an AOT reverse micelle, these metrics are particularly well suited to topological balls. This rough group would include all possible types of micelles (provided the entire interior is considered part of the system), but also systems such as bacteria and other single celled organisms, liquid-in-gas droplets or gas-in-liquid bubbles, globular proteins, and nanoparticles. While proteins and nanoparticles are typically well defined and therefore not obvious candidates for metrics characterizing amorphous shapes, characterizing the exact shape may not be easy or irregularities such as crystal defects may present challenges. Therefore, it may be preferable in certain circumstances to use the metrics presented here to roughly characterize a well-defined protein, or to provide a better measure for when a protein transitions from one conformation to another. It may also be preferable to use these metrics to characterize the real shape of individual nanoparticles, including the crystal defects. These metrics could also be reasonably applied to long tubes with relative ease such as the hydrophilic cavities within a Nafion membrane, the shape of a single strand of a polymer, or the shape of long aggregates such as carbon nanotube ropes or tube-shaped self-assembled objects. ${ }^{10}$ The study of shapes other than topological balls presents a challenge. This includes topological spheres such as a cell membrane that does not include the interior of the cell, or our own micelles if we removed the water from consideration. It could also include shapes such as those observed in the work of Vasquez, et al., whose simulation of an AOT reverse micelle has apparently formed a toroidal shape. ${ }^{33}$ While this greatly intrigues us and begs further study, in this case, our definition of convexity, in particular, would be challenged because the volume relative to the convex hull would be dramatically reduced by the cavity and convexity would not be responsive to changes in the interior surface.

We encourage readers hoping to apply our metrics to carefully consider their specific system and modify our metrics as needed to adequately describe that system. For example, in the case of the hollow cavity, a new definition of convexity might need to be explored, such as a definition based on surface area rather than volume. Alternatively, it could be 
advantageous to consider the interior and exterior surfaces separately and track them independently; although it could also be just as good to simply use the definitions we have presented and simply ignore the interior cavity, depending on what the requirements and interests of that particular system are. We cannot answer to all hypotheticals in a single paper and the reader is advised to take such things into consideration. However, we are confident that the majority of real systems which might be encountered in chemistry will allow the metrics presented here to be used without modification and we hope this serves as an adequate framework for developing new measurements in the rare cases in which these metrics fail or are insufficient.

\section{Acknowledgement}

We gratefully acknowledge financial support from Colorado State University and NSF Grant 1956323. This work utilized the resources from the University of Colorado Boulder Research Computing Group, which is supported by the National Science Foundation (awards ACI-1532235 and ACI-1532236), the University of Colorado Boulder, and Colorado State University. We would like to thank Drs. Joseph DiVerdi, Grzegorz Szamel, and Chris Petersen for reviewing the manuscript and their exceptionally helpful comments.

\section{Supporting Information Available}

The following files are available free of charge:

- Rotating GIF's of all micelles shown in the paper, to provide a 3D view of these frames

- The code used in this paper is provided as a public GitHub repository. https:// github.com/Levinger-Group/How-to-think-about-Shape

- A Supporting Information document providing several extra figures and notes to further explain various ideas presented in the paper. 


\section{References}

(1) Dalton, J. A new system of chemical philosophy; S. Russell: London, 1808.

(2) Historical Profile: August Kekulé and Archibald Scott Couper. 2017; https://www. sciencehistory.org/historical-profile/.

(3) Robinson, A. Chemistry's visual origins. Nature 2010, 465, 36.

(4) Rocke, A. J. Image and Reality: Kekule, Kopp, and the Scientific Imagination; University of Chicago Press, 2010; p 416.

(5) Ashdown, A. A. Earliest History of the Friedel-Crafts Reaction. Ind. Eng. Chem. 1927, 19, 1063-1065.

(6) Ferry, G. History: Women in crystallography. Nature 2014, 505, 609-611.

(7) Hodgkin, D. C. The X-ray analysis of the structure of penicillin. Adv. Sci. 1949, 6, $85-89$.

(8) Demain, A.; Elander, R. The beta-lactam antibiotics: past, present, and future. Antonie van Leeuwenhoek 1999, 75, 5-19.

(9) Howard, J. A. K. Dorothy Hodgkin and her contributions to biochemistry. Nat. Rev. Mol. Cell Biol. 2003, 4, 891-896.

(10) Kundu, S.; Chowdhury, A.; Nandi, S.; Bhattacharyya, K.; Patra, A. Deciphering the evolution of supramolecular nanofibers in solution and solid-state: a combined microscopic and spectroscopic approach. Chem. Sci. 2021, 12, 5874-5882.

(11) Levinger, N. E. Water in confinement. Science 2002, 298, 1722-1723.

(12) Harpham, M. R.; Ladanyi, B. M.; Levinger, N. E. The effect of the counterion on water mobility in reverse micelles studied by molecular dynamics simulations. J. Phys. Chem. B 2005, 109, 16891-16900. 
(13) Piletic, I. R.; Moilanen, D. E.; Spry, D. B.; Levinger, N. E.; Payer, M. D. Testing the core/shell model of nanoconfined water in reverse micelles using linear and nonlinear IR spectroscopy. Journal of Physical Chemistry A 2006, 110, 4985-4999.

(14) Park, S.; Moilanen, D. E.; Fayer, M. D. Water dynamics-The effects of ions and nanoconfinement. J. Phys. Chem. B 2008, 112, 5279-5290.

(15) Wiebenga-Sanford, B. P.; Washington, J. B.; Cosgrove, B.; Palomares, E. F.; Vasquez, D. A.; Rithner, C. D.; Levinger, N. E. Sweet Confinement: Glucose and Carbohydrate Osmolytes in Reverse Micelles. J. Phys. Chem. B 2018, 122, 9555-9566.

(16) Miller, S. L.; Wiebenga-Sanford, B. P.; Rithner, C. D.; Levinger, N. E. Nanoconfinement Raises the Energy Barrier to Hydrogen Atom Exchange between Water and Glucose. J. Phys. Chem. B 2021, 125, 3364-3373.

(17) Luisi, P. L.; Giomini, M.; Pileni, M. P.; Robinson, B. H. Reverse micelles as hosts for proteins and small molecules. BBA 1988, 947, 209-246.

(18) Lye, G. J.; Asenjo, J. A.; Pyle, D. L. Extraction of lysozyme and ribonuclease-a using reverse micelles: Limits to protein solubilization. Biotechnol Bioeng. 1995, 47, 509-519.

(19) Melo, E. P.; Aires-Barros, M. R.; Cabral, J. M. Biotechnology Annual Review; 2001; Vol. 7; pp 87-129.

(20) Bagwe, R. P.; Khilar, K. C. Effects of the intermicellar exchange rate and cations on the size of silver chloride nanoparticles formed in reverse micelles of AOT. Langmuir 1997, 13, 6432-6438.

(21) Cason, J. P.; Roberts, C. B. Metallic copper nanoparticle synthesis in AOT reverse micelles in compressed propane and supercritical ethane solutions. J. Phys. Chem. B 2000, 104, 1217-1221. 
(22) Qian, Y.; Wen, W.; Adcock, P. A.; Jiang, Z.; Hakim, N.; Saha, M. S.; Mukerjee, S. $\mathrm{PtM} / \mathrm{C}$ catalyst prepared using reverse micelle method for oxygen reduction reaction in PEM fuel cells. J. Phys. Chem. C 2008, 112, 1146-1157.

(23) Assih, T.; Larché, F.; Delord, P. Evolution of the radius of the inverse micelles at high dilution in the aerosol-OT/Water/n-decane system. J. Colloid Interface Sci. 1982, 89, $35-39$.

(24) Pileni, M. P.; Zemb, T.; Petit, C. Solubilization by reverse micelles: Solute localization and structure perturbation. Chem. Phys. Lett. 1985, 118, 414-420.

(25) Kotlarchyk, M.; Huang, J. S.; Chen, S.-H. Structure of AOT Reversed Micelles Determined by Small-Angle Neutron Scattering. J. Phys. Chem 1985, 89, 4382-4386.

(26) Quist, P. O.; Halle, B. Water dynamics and aggregate structure in reversed micelles at sub-zero temperatures. A deuteron spin relaxation study. J. Chem. Soc. Faraday Trans. 1 1988, 84, 1033-1046.

(27) Onori, G.; Santucci, A. Viscosity studies of water-containing reversed aot micelles. J. Colloid Interface Sci. 1992, 150, 195-199.

(28) Nave, S.; Eastoe, J.; Penfold, J. What is So Special about Aerosol-OT? 1. Aqueous Systems. Langmuir 2000, 16, 8733-8740.

(29) Balakrishnan, S.; Javid, N.; Weingärtner, H.; Winter, R. Small-angle X-ray scattering and near-infrared vibrational spectroscopy of water confined in aerosol-OT reverse micelles. ChemPhysChem 2008, 9, 2794-2801.

(30) Sheu, E. Y.; Chen, S.-H. Structure and Growth of Bis(2-ethylhexyl) Sulfosucclnate Micelles. J. Phys. Chem. 1987, 91, 3306-3310.

(31) Giordano, R.; Migliardo, P.; Wanderlingh, U.; Bardez, E.; Vasi, C. Structural properties of micellar solutions. J. Mol. Struct. 1993, 296, 265-269. 
(32) Bohidar, H. B.; Behboudnia, M. Characterization of reverse micelles by dynamic light scattering. Colloid Surf. A 2001, 178, 313-323.

(33) Vasquez, V. R.; Williams, B. C.; Graeve, O. A. Stability and Comparative Analysis of AOT/Water/Isooctane Reverse Micelle System Using Dynamic Light Scattering and Molecular Dynamics. J. Phys. Chem. B 2011, 115, 2979-2987.

(34) Faeder, J.; Ladanyi, B. M. Molecular Dynamics Simulations of the Interior of Aqueous Reverse Micelles. J. Phys. Chem. B 2000, 104, 1033-1046.

(35) Abel, S.; Sterpone, F.; Bandyopadhyay, S.; Marchi, M. Molecular modeling and simulations of AOT-water reverse micelles in isooctane: Structural and dynamic properties. J. Phys. Chem. B 2004, 108, 19458-19466.

(36) Chowdhary, J.; Ladanyi, B. M. Molecular dynamics simulation of aerosol-OT reverse micelles. J. Phys. Chem. B 2009, 113, 15029-15039.

(37) Martinez, A. V.; Dominguez, L.; Małolepsza, E.; Moser, A.; Ziegler, Z.; Straub, J. E. Probing the structure and dynamics of confined water in AOT reverse micelles. J. Phys. Chem. B 2013, 117, 7345-7351.

(38) Marchi, M.; Abel, S. Modeling the self-aggregation of small AOT reverse micelles from first-principles. J. Phys. Chem. Lett 2015, 6, 170-174.

(39) Urano, R.; Pantelopulos, G. A.; Song, S.; Straub, J. E. Characterization of dynamics and mechanism in the self-assembly of AOT reverse micelles. J. Chem. Phys. 2018, 149

(40) Nevidimov, A. V.; Razumov, V. F. Molecular dynamics simulations of AOT reverse micelles' self-assembly. Mol. Phys. 2009, 107, 2169-2180.

(41) Eskici, G.; Axelsen, P. H. The size of AOT reverse micelles. J. Phys. Chem. B 2016, 120, 11337-11347. 
(42) Zhou, B.; Wang, J.; Zhao, B. Micromorphology characterization and reconstruction of sand particles using micro X-ray tomography and spherical harmonics. Eng. Geol. 2015, 184, 126-137.

(43) Garboczi, E. J.; Bullard, J. W. 3D analytical mathematical models of random starshape particles via a combination of X-ray computed microtomography and spherical harmonic analysis. Adv. Powder Technol. 2017, 28, 325-339.

(44) Abel, S. Stéphane Abel's Homepage, Downloads, Equilibrated AOT Reverse Micelle. 2011; http://st-abel.com/downloads.htm.

(45) Maitra, A. Determination of size parameters of water-aerosol OT-oil reverse micelles from their nuclear magnetic resonance data. J. Phys. Chem. 1984, 88, 5122-5125.

(46) Klauda, J. B.; Venable, R. M.; Freites, J. A.; O'Connor, J. W.; Tobias, D. J.; Mondragon-Ramirez, C.; Vorobyov, I.; MacKerell, A. D.; Pastor, R. W. Update of the CHARMM All-Atom Additive Force Field for Lipids: Validation on Six Lipid Types. J. Phys. Chem. B 2010, 114, 7830-7843.

(47) Jorgensen, W. L.; Chandrasekhar, J.; Madura, J. D.; Impey, R. W.; Klein, M. L. Comparison of simple potential functions for simulating liquid water. J. Chem. Phys. 1983, $79,926-935$.

(48) Martínez, L.; Andrade, R.; Birgin, E. G.; Martínez, J. M. PACKMOL: A Package for Building Initial Configurations for Molecular Dynamics Simulations. J. Comput. Chem. 2009, 30, 2157-2164.

(49) Berendsen, H. J.; van der Spoel, D.; van Drunen, R. GROMACS: A message-passing parallel molecular dynamics implementation. Comp. Phys. Comm. 1995, 91, 43-56.

(50) Van Der Spoel, D.; Lindahl, E.; Hess, B.; Groenhof, G.; Mark, A. E.; Berendsen, H. J. GROMACS: Fast, flexible, and free. J. Comp. Chem. 2005, 26, 1701-1718. 
(51) Abraham, M. J.; Murtola, T.; Schulz, R.; Páll, S.; Smith, J. C.; Hess, B.; Lindah, E. Gromacs: High performance molecular simulations through multi-level parallelism from laptops to supercomputers. SoftwareX 2015, 1-2, 19-25.

(52) Du, C.; He, W.; Yin, T.; Shen, W. Volumetric properties of water/AOT/isooctane microemulsions. Langmuir 2014, 30, 15135-15142.

(53) Bussi, G.; Donadio, D.; Parrinello, M. Canonical sampling through velocity rescaling. J. Chem. Phys. 2007, 126.

(54) Berendsen, H. J.; Postma, J. P.; Van Gunsteren, W. F.; Dinola, A.; Haak, J. R. Molecular dynamics with coupling to an external bath. J. Chem. Phys. 1984, 81, 3684-3690.

(55) Hoover, W. G. Canonical dynamics: Equilibrium phase-space distribution. Phys. Rev. A 1985, 31, 1695-1697.

(56) Nosé, S. A molecular dynamics method for simulations in the canonical ensemble. Mol. Phys. 2002, 100, 191-198.

(57) Parrinello, M.; Rahman, A. Polymorphic transitions in single crystals: A new molecular dynamics method. J. Appl. Phys. 1981, 52, 7182-7190.

(58) Nosé, S.; Klein, M. Constant pressure molecular dynamics for molecular systems. Mol. Phys. 1983, 50, 1055-1076.

(59) Willard, A. P.; Chandler, D. Instantaneous Liquid Interfaces. J. Phys. Chem. B 2010, 114, 1954-1958.

(60) Sega, M.; Hantal, G.; Fábián, B.; Jedlovszky, P. Pytim: A python package for the interfacial analysis of molecular simulations. J. Comput. Chem. 2018, 39, 2118-2125.

(61) Barber, C. B.; Dobkin, D. P.; Huhdanpaa, H. The Quickhull Algorithm for Convex Hulls. ACM Trans. Math. Softw. 1996, 22, 469-483. 
(62) Michaud-Agrawal, N.; Denning, E. J.; Woolf, T. B.; Beckstein, O. MDAnalysis: a Toolkit for the Analysis of Molecular Dynamics Simulations. Journal of computational chemistry 2011, 32, 2319-2327.

(63) Gowers, R. J.; Linke, M.; Barnoud, J.; Reddy, T. J.; Melo, M. N.; Seyler, S. L.; Domański, J.; Dotson, D. L.; Buchoux, S.; Kenney, I. M. et al. MDAnalysis: A Python Package for the Rapid Analysis of Molecular Dynamics Simulations. Proceedings of the 15th Python in Science Conference. 2016; pp 98-105.

(64) Sullivan, C. B.; Kaszynski, A. PyVista: 3D plotting and mesh analysis through a streamlined interface for the Visualization Toolkit (VTK). J. Open Source Softw. 2019, 4, 1450 .

(65) Ayoub, A. B. The Eccentricity of a Conic Section. Coll. Math. J. 2003, 34, 116-121.

(66) Weisstein, E. W. Eccentricity. https://mathworld.wolfram.com/Eccentricity. html.

(67) Weisstein, E. W. Moment of Inertia. https://mathworld.wolfram.com/ MomentofInertia.html.

(68) Weisstein, E. W. Ellipsoid. https://mathworld.wolfram.com/Ellipsoid.html.

(69) Žunić, J.; Rosin, P. A Convexity Measurement for Polygons. Proceedings of the British Machine Vision Conference. 2002; pp 173-182.

(70) Kreyszig, E. Differential Geometry; Dover Publications: New York, 1991; Chapter Chapter II, pp 34-36.

(71) Gray, A. Modern Differential Geometry of Curves and Surfaces with Mathematica, 2nd ed.; CRC Press: Boca Raton, 1997; Chapter Metrics on Surfaces, pp 363-367.

(72) Gray, A. Modern Differential Geometry of Curves and Surfaces with Mathematica, 2nd ed.; CRC Press: Boca Raton, 1997; Chapter The Theory, pp 373-380. 
(73) Allen, R.; Bandyopadhyay, S.; Klein, M. L. C12E2 reverse micelle: A Molecular Dynamics Study. Langmuir 2000, 16, 10574-10552.

(74) Salaniwal, S.; Cui, S.; Cochran, H. D.; Cummings, P. T. Molecular dynamics simulation of reverse micelles in supercritical carbon dioxide. Ind. Eng. Chem. Res. 2000, 39, $4543-4554$.

(75) Cromwell, P. R. Polyhedra; Cambridge University Press: New York, 1997.

(76) Weisstein, E. W. Augmentation. https://mathworld.wolfram.com/Augmentation. html. 


\section{TOC Graphic}

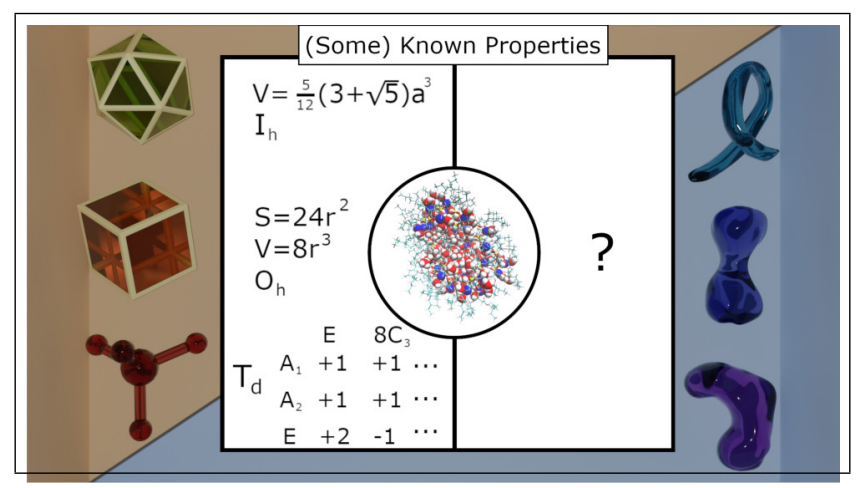

